\title{
Is Information a Sufficient Basis for Cognition? Part 2: Physical Foundations
}

\author{
José María Díaz Nafría*, Mario Pérez-Montoro** \\ * jdian@unileon.es, Science of Information Institute, Washington, U.S.A; Universidad de León, Spain \\ *** perez-montoro@ub.edu, Department of Information Science, University of Barcelona, Spain
}

\begin{abstract}
In this second part of our inquiry into the relation between information and cognition, we delve into the physical limits of the manifestation of an arbitrary object first with independence of any observer, then considering the nature of perception. The analysis of the manifestations of an object in a homogeneous environment by means of wave phenomena shows that the information carried by such manifestations offers a constitutive fuzziness and ambiguity of the observed object. On the one hand, the details that can be specified concerning the object are strictly limited by the wave length; on the other hand, the volumetric details of the object (i.e. its bowls) are outlawed to the observer, not in virtue of the object opacity, but to the very dimension or complexity of the wave phenomenon in the space surrounding the object. The analysis of perception, considering this physical boundary and the specificity of the animal sensitivity, shows the combined role of other concurrent or previous percept and some a priori knowledge in the perception and awareness of reality.
\end{abstract}

Keywords: Physics of observation, Waves, Inverse problem, Theory of perception, Biophysics, Epistemology, Cognition

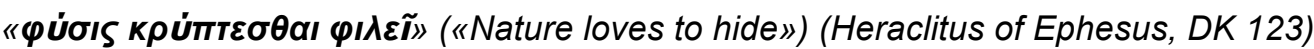

\section{The Nature of the Manifestation of Reality and Rerception}

When an observer puts attention into the manifestations of an object, no matter if they are due to the mechanical or electromagnetic interactions with the environment (as respectively are sound and light), the observing properties of such an environment respond -where the observer is located- to the well-known wave equation:

$$
\nabla^{2} \Psi(r, t)=\frac{1}{v^{2}} \frac{\partial^{2} \Psi(r, t)}{\partial t^{2}}
$$

where $\boldsymbol{\Psi}$ represents the properties of the environment to which the observed is allegedly sensitive (e.g. the air pressure or the light), $\mathbf{r}$ is the position vector, $t$ the temporal variable, and $v$ a constant depending on the environment characteristics and corresponding to the propagation speed of the wave phenomenon. The pertinence of (1) implies that the observation domain (or domain of manifestation of the object) is homogeneous and isotropic (i.e., the interactions depend on neither the direction nor the position in which the parts are located).

In Figure 1 is explained, if there is an external illumination by means of a plane wave (i.e. far away situated focus) the radiation reaching the observer will be the sum of a plane wave and the wave scattered by the object. Spatially, the scattered wave will present the most differences, and these will correspond to the superficial qualities of the object, which can be explored by the observation. Therefore, the main part of what might be branded as scattering problem can be interpreted as if the object were the very source of radiation. 


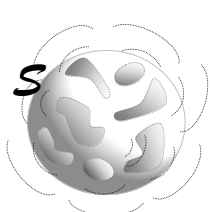

Object

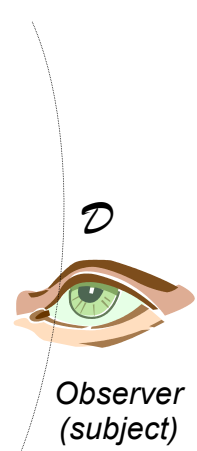

(subject)

Figure 1: Scheme of observation of an object generating a wave phenomenon.

The relative complexity of (1) may be smoothed if, by means of the Fourier theorem, any temporal variation is expressed as a linear combination of harmonic ones. Thus, we can separate a relation for each involved frequency, $f$ (and a later combination of single-frequency variations may render the full temporal evolution), namely the Helmholtz equation:

$$
\nabla^{2} \Psi(r)+k^{2} \Psi(r)=0
$$

where the wave number $k=2 \pi f / v=2 \pi / \lambda$, being $\lambda$ the wavelength for the involved frequency, and the complex values of $\boldsymbol{\Psi}$ reflect the amplitude and phase of the $f$ component of the temporal phenomenon

$$
\Psi(r, t)=\int_{\forall \in \in B} \Re\left\{\Psi_{f}(r) e^{-i 2 \pi f t}\right\} d f
$$

If we now apply the Fourier Theorem again in the spatial dimensions, we can find a linear combination of harmonic variations in each of the spatial directions which allow us to represent any spatial distribution of the observed property $\boldsymbol{\Psi}$. Thus, it can be expressed as a linear combination of distributions of the following type:

$$
\boldsymbol{\Psi}(\mathbf{r})=\boldsymbol{\Psi}_{x}(\mathbf{r}) \cdot \boldsymbol{\Psi}_{y}(\mathbf{r}) \cdot \boldsymbol{\Psi}_{z}(\mathbf{r}) \text { with } \boldsymbol{\Psi}_{u}=\mathbf{A}_{u} e^{-i k_{u} u}+\mathbf{A}_{-u} e^{i k_{u} u}
$$

where $u$ represents any spatial direction, and $k_{u}$ its corresponding spatial frequency or the wavenumber in the direction $u{ }^{1}$ Now applying (3) to ec. (2), we obtain:

$$
k_{x}^{2}+k_{y}^{2}+k_{z}^{2}=k^{2}
$$

These wave-numbers for each spatial direction might be interpreted in terms of components of spatial frequency components (i.e. sinusoidal distributions in the corresponding spatial direction).

\footnotetext{
${ }^{1}$ If ku is limited to real values, it is clear that a valid type of solution for ec. (2) is not considered, corresponding to exponentially decaying distributions, named evanescent modes. Therefore, (3) and the following discussion just refer to harmonic distributions. However, we should stress that although the absence of evanescent modes is not strictly the case (i.e. there are indeed evanescent waves around the object) in a practical sense such waves do not go beyond the very vicinity of the object in an observable level. That is, its actual level may be underneath noise level or observer sensibility.
} 
Hence, relation (4) implies that the harmonic variations that may be expected in each direction are limited to a fundamental constraint, which may be geometrically expressed as a spherical surface (Fig.2). The shaded area corresponds to the special frequencies expected in a plane (constant $z$ ). The spatial frequencies in the $\mathrm{x}$ and $\mathrm{y}$ direction within the shaded area are named visible (spatial) spectrum, the outer points correspond to evanescent waves, which only manifest in the close vicinity of the object.

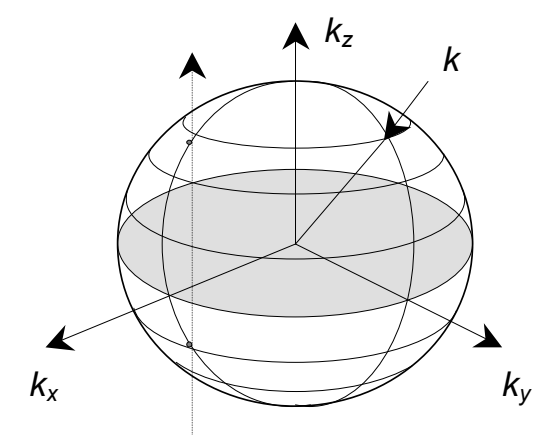

Figure 2: Surface of spatial frequencies, isomorphic with the domain of fields generated in the homogeneous space.

\section{The Limits of Observation}

\subsection{Discretizability of the Observable Phenomena}

Assuming the geometric representation of ec. (4), we should admit that the maximal spatial frequency expected in any direction is $k$ (expressed in number of angular waves, $\mathrm{rad} / \mathrm{m}$ ) or $k / 2 \pi$ (expressed in $1 / \mathrm{m}$, which we will name $b$ ). That is, the spatial observable distribution is strictly bandlimited.

Theorem 1: The minimal distance between independent intensities values of a field generated by an arbitrary object is $\lambda / 2$.

According to (4) it is clear that the maximal spatial frequency in any direction is $k$. Thus, according to the sampling theorem, the spatial distribution is completely determined by the values distanced $1 / 2 b=1 /(2 k / 2 \pi)=\pi / k$ (Shannon, 1949, p.11). Hence, any value of the real distribution in-between can be obtained by the set of values maintaining such separation. Therefore, these values inbetween can be considered as dependent. In other words, the minimal distance between independent values of a field is strictly $\pi / k=\pi \lambda / 2 \pi=\lambda / 2$, as we had stated.

In other words, no matter how much we sharpen our seeing, there is a maximum limit of details we might observe independently of where we are located. Nevertheless, if we approach much to the object, it might happen that the -observable- presence of evanescent modes increases. To take it into account, an special bandwidth excess factor $x>1$ will be sufficient. A correct election will allow us to make an optimal sampling over a surface embracing the observed object, $s$ (s. fig. 1 ). In this case, the minimal separation between independent values is $\lambda / 2 X$.

This discretizability of the observable phenomenon implies that the apparent continuous distribution problem is indeed reduced to another problem of discrete distribution, whose dimension is infinitely smaller, or versus the dimension innumerability of an arbitrary continuous distribution (determined by the values at every point of the continuous), the observation problem dimension is now numerable. 


\subsection{Dimensionality of the Observing Problem}

If we now consider that the uniqueness of solution of the Helmholtz equation over all the homogeneous space is guaranteed by the knowledge of the field distribution over any surface enclosing the observed object (for instance, the bounding surface $S$ ), hence, we may speak about a maximum number of observable details -from any observation domain $\mathcal{D}$ - corresponding to the object. This number can be regarded as the dimension of the observation problem (or number of unknowns previous to an observation for a given size of the object). ${ }^{2}$

Theorem 2: The maximum number of details of an object, inscribed in an sphere of radius a, which is causing an observed field distribution is $16 \pi(a \chi \mid \lambda)^{2}$. This is the essential dimension of the observation problem.

Considering -as aforementioned- that the minimum distance between independent samples is $\lambda / 2 \chi$, and the area of the domain $s$ enclosing the object is $4 \pi a^{2}$, thus we can speak about a finite number $N$ of independent sub-domains (characterised by only a sample) of area $(\lambda / 2 \chi)^{2}$. Such independence implies that: $1^{\text {st }}$ ) according to theorem 1, the field distribution over all $S$ can be determined by such samples, and $2^{\text {nd }}$ ) according to the uniqueness theorem, the field at any other place of the homogeneous space of manifestation of the object can also be determined (Colton \& Kress, 1998; Díaz, 2003, §2.2). Consequently, the samples of the field at the $N$ independent sub-domains over the minimal ball enclosing the object allows to completely determine the field generated around the domain $S$. Therefore, $N$ corresponds to the dimension of the observation problem (namely, essential dimension), where $N=$ (area of $S$ ) / (area of an independent sub-domain) $=$ $4 \pi a^{2} /(\lambda / 2 \chi)^{2}$, as we had stated.

\subsection{Discretizability of a Distant Observation}

As we have previously shown, the minimal distance between independent values of the field distribution is $\lambda / 2$-theorem 1-, however, as we have just seen the dimension of the observation problem is finite; thus, it is to expect that by increasing the distance to the object, the spatial concentration of observable differences will decrease.

Theorem 3: The minimal distance between independent values of the field corresponding to the manifestation of an object inscribed in a sphere of radius a, whose centre is at a distance $d$, is: $\lambda d / 2 a x$.

If the field distribution is described by spherical functions ${ }^{3}$, it can be observed despite the order of the function (concerning its properties with respect to distances further away than the wavelength) that all of them present the same radial decay. From this property it can be inferred that at different distances away from the object the same angular variations are expected. Thus, if we observe over a curve of radius $r$ centred in the origin, the distance between independent angles of observation will be: $(\lambda / 2 X) / r=\lambda / 2 r x$. On the other hand, if we consider that the Helmholtz equation is only satisfied in the homogeneous space (therefore out of the observed object, $r>a$ ), the distance between independent observation angles will be $\lambda / 2 a x$. Hence, the distance between independent values of the field distribution observed at a distance $d$ will be: $\lambda d / 2 a x$, as we had stated.

\footnotetext{
${ }^{2}$ Strictly, the uniqueness depends on the realistic assumption that the radiating field -coming from the object under observation- tends to cancel with the distance. This and other assumptions -in acoustics, electromagnetic radiation, etc- are generally acknowledged as uniqueness theorems (Díaz, 2003, pp. 20, 87, 123; Colton \& Kress 1998, §5.1, 7.1).

${ }^{3}$ Observing a field distribution over a spherical surface, the distribution can be expressed as a linear combination of spherical functions, which are solutions of the Helmholtz equation (2) expressed in spherical coordinates (Weisstein, 2008).
} 


\subsection{The Forward and the Inverse Problems}

We might still complete our characterization of the observation problem to refer, for instance, how to determine the excess factor $X$ (it is here enough to notice that for objects whose maximal dimension is smaller than $2 \lambda, x$ can be made approximately 1 ); how to elect the independent subdomains for non planar surfaces; or how to determine the values of the field distribution over any other point of the domain of observation, $\mathcal{D}$, of the domain enclosing the object, $S$, or any other at the surrounding homogeneous space. ${ }^{4}$ All these are fundamental problems for a detailed statement of the forward problem (i.e. finding the manifestations of an object which is well known) and for a complete formulation of the inverse problem (i.e. finding the causes, or description of the object, originating the observed phenomenon). However they are not essential for discussing the limits of the observation, as we intend.

Suffice it to say that on the basis of the former theorems we can show that independently of the volumetric distribution of the sources we are intending to know (observation object) the expression of the forward problem can take the form of a linear transformation between the sources and the observed field. This transformation, thanks to the discretizability previously shown, can be expressed by means of the following matrix relation:

$$
\left(\begin{array}{c}
\Psi\left(u_{1}, v_{1}\right) \\
\vdots \\
\Psi\left(u_{M}, v_{M}\right)
\end{array}\right)=\left(\begin{array}{ccc}
G\left(u_{1}, v_{1}, x_{1}^{\prime}, y_{1}^{\prime}, z_{1}^{\prime}\right) & \cdots & G\left(u_{1}, v_{1}, x_{N}^{\prime}, y_{N}^{\prime}, z_{N}^{\prime}\right) \\
\vdots & \ddots & \vdots \\
G\left(u_{M}, v_{M}, x_{1}^{\prime}, y_{1}^{\prime}, z_{1}^{\prime}\right) & \cdots & G\left(u_{M}, v_{M}, x_{N}^{\prime}, y_{N}^{\prime}, z_{N}^{\prime}\right)
\end{array}\right) \times\left(\begin{array}{c}
f\left(x_{1}^{\prime}, y_{1}^{\prime}, z_{1}^{\prime}\right) \\
\vdots \\
f\left(x_{N}^{\prime}, y_{N}^{\prime}, z_{N}^{\prime}\right)
\end{array}\right)
$$

where $(u, v)$ represent curvilinear coordinates over the observation space; $1 \ldots N$ the cardinals of the samples over the observation domain; $\left(x^{\prime}, y^{\prime}, z^{\prime}\right)$ the locations of the founts or sources -i.e. the object- properly discretized; $1 \ldots M$ the cardinals of these samples; and $G\left(u, v, x^{\prime}, y^{\prime}, z^{\prime}\right)$ the Green function, which at the same time satisfies the wave equation (2) in the homogeneous space and establishes a direct relation to the non-homogeneity, to which the presence of the object (or one of its infinitesimal parts) intrinsically responds: between the location $\left(x^{\prime}, y^{\prime}, z^{\prime}\right)$ at the sources and $(u, v)$ at the observation domain.

More densely, we can express the relation between field and its corresponding sources grouping the field distribution into a $M$-dimensional vector $\boldsymbol{\Psi}$, the sources into a $N$-dimensional vector $f$, and the transformation among them by a matrix operator $\mathcal{T}$.

$$
\boldsymbol{\Psi}=\left(\begin{array}{c}
\Psi_{1} \\
\vdots \\
\Psi_{M}
\end{array}\right)=\sum_{n=1}^{N}\left(\begin{array}{c}
\psi_{1} \\
\vdots \\
\psi_{M}
\end{array}\right) \cdot f_{n}=\sum_{n=1}^{N} \boldsymbol{\Psi}_{n} \cdot f_{n}=\mathcal{T} \cdot f \quad \text { where } \boldsymbol{\Psi}_{n}=\left(\begin{array}{c}
G\left(u_{1}, v_{1}, x_{n}^{\prime}, y_{n}^{\prime}, z_{n}^{\prime}\right) \\
\vdots \\
G\left(u_{M}, v_{M}, x_{n}^{\prime}, y_{n}^{\prime}, z_{n}^{\prime}\right)
\end{array}\right)
$$

which can be interpreted as the wave function for each discrete source of unitary amplitude.

The forward problem does not have any difficulty: if we indeed knew the distribution of sources, described in terms of $f$, it would suffice to apply the former relations to know how actually the manifestations in terms of $\boldsymbol{\Psi}$ are. We do not care if $N$ is bigger or smaller. However, this is -so to speaka pseudo-problem if we give for granted the way the environment transmits the changes in one part of the space (in our case, the validity of the wave equation in our real environment), and of course, it does not correspond to the observation problem as we have stated it. Generally, our knowledge about the objects is not a priori but a posteriori, that is, reckoning with its manifestation. This is the inverse problem, which in our formulation implies obtaining $f$ from $\Psi$. In this case, it is evident that the dimension $N$ is important, since we would never invert the relation if $N>M$. It is also important the independency of the wave functions $\boldsymbol{\Psi}_{n}$, or at least, that the dimensionality (or complexity) of

\footnotetext{
${ }^{4}$ For a discussion of these and further questions concerning the inverse problem cf. the author's work (Díaz, 2003).
} 
the space developed by an arbitrary set of $N$ sources corresponds to the dimensionality (or complexity) of an arbitrary observation.

\subsection{The Inverse Problem}

Theorems 2 and 3 establish fundamental limits which enable a proper arrangement of our problem: according to theorem 3, the actual dimension of the observed phenomenon does not depend on how detailed the observation is, since we often have to move quite distant to find some independent value of the considered phenomenon. The number of spatial details that we can perceive can never be higher than the essential dimension predicted by theorem 2. Thus, it is also the maximal number of details that might be specified concerning the object. It is here relevant to remind that such dimension does not depend on the volume $\left(\propto a^{3}\right)$ but on the bounding surface $\left(\propto a^{3}\right)$, which let us arrive to a fundamental conclusion: the volumetric distribution of the object is inscrutable. In this case, what might we know about the object?

At this point, we should remember the Huygens principle (1690). It establishes that "each point on a primary wavefront can be considered to be a new source of a secondary spherical wave and that a secondary wavefront can be constructed as the envelope of these secondary spherical waves." It suffices to refer to the secondary sources (also considered as "equivalent sources") distributed on the surface bounding the object, which is properly located at the homogeneous space. As we have just shown, the dimensionality of the observation and that of the radiated field around the object implies that we can only obtain from the object a superficial knowledge, which can be interpreted as a projection of what is inside. But it is forbidden to come into that "inside", just based on a posteriori knowledge.

To clarify this last condition, we must take into account that if the inner complexity of the object structure is smaller than the essential dimension $N$, then the observer could grasp an idea of the volumetric distribution. Now, such 'idea' would be achieved based on an assumption of inner structure, since there is in principle an unlimited number of inner structures whose projections over a bounding surface are equal.

If we also take into account the dimensional limitation at the source domain (theorem 2) and the separation required for the independence of the equivalent sources at the bounding surface (here translated into the independence of the fields generated over the observation domain, 0 ): a good way to suit our problem is locating punctual sources over $S$ regularly spaced at a distance $\lambda / 2 X$. The space of radiated fields which could be generated by this discrete distribution of equivalent punctual sources over $s$ is equivalent to the one that could be generated by any inner (discrete or continuous) volumetric distribution. It can be shown (Díaz, 2003, §3.2.1) that if a quadratic norm is defined for the mentioned space of radiated fields as well as a distance between field distributions based on this norm $d\left(\boldsymbol{\Psi}_{\mathrm{i}}, \boldsymbol{\Psi}_{\mathrm{j}}\right)$, then there will only be a unique distribution of equivalent punctual sources over $\boldsymbol{S}$, which can be considered as an orthogonal projection of the observed field $\boldsymbol{\Psi}$ on the source domain $f$ :

$$
\boldsymbol{\Psi}=\left\{\begin{array}{c}
\boldsymbol{\Psi}_{\text {OBSERVED }} \\
\mathcal{T} \cdot f_{\text {projection }}
\end{array}\right\} \Rightarrow \exists f_{\text {projection }}=\left[\mathcal{T}^{+} \cdot \mathcal{T}\right]^{-1} \mathcal{T}^{+} \cdot \boldsymbol{\Psi}_{\text {OBS }} / \min _{f}\left\{q\left(\mathcal{T} \cdot f_{\text {projection }}, \boldsymbol{\Psi}_{\text {OBS. }}\right)\right\}
$$

where $\mathcal{T}^{\star}$ represents the adjoint matrix of $\mathcal{T}$.

\footnotetext{
${ }^{5}$ This principle can be justified by means of the aforementioned uniqueness theorem (see $\S 2.2$ and note 2), which for the electromagnetic problem was rigorously stated by Schelkunoff in terms of the equivalence theorem (Schelkunoff, 1936).
} 


\subsection{Observational Limits and Perception}

Based upon the previous theorems, the following fundamental conclusions can be forward extracted, which concern what can be known about the object causing an observed wave phenomenon:

- The number of details to be found in the environment due to the presence of the object is finite.

- Such number depends on the surface bounding the object and not on its volume.

- The volumetric distribution of an object cannot be known only based on its manifestations on the environment.

- The description of the object that can be achieved corresponds to a projection of the inner inhomogeneities over a bounding surface.

These four conclusions establish fundamental limits to the observation problem, not attached to the specificity of our organs of animal or human sensibility, but to the differences that can merely be found in the environment and the maximal knowledge that could be derived concerning the object causing these differences. Using Kantian terminology, these are the limits in the determination of an object of knowledge by means of a transcendental subject, to which the intimate knowledge of the object is forbidden -as we previously showed-. In other words, in spite of the actual complexity of the object, the complexity of the manifestations in the space surrounding the object -due to its presence- is constitutively smaller than the complexity of the object. We could argue that this were the case unless the object could be completely described in its projection over the bounding surface. But even in this case, the observation does not suffice to conclude that this completeness is the case, we must also know for instance that the inner part is empty, since there is a whole set of possibilities regarding the inner configuration. As previously pointed out, another possibility for a complete determination is that the observer intends to find out the specific configuration of a structure whose degrees of freedom are equal or smaller than the complexity of the field in the surrounding space, which obviously implies an important amount of previous knowledge.

Our analysis could be considered trivial if we just think in its correspondence to the visual problem, since there is a danger to confuse the limits to acknowledge the inner part of an object with its opacity. Even if some degree of transparency could be ascribed to all the inner parts, the limit concerning the complexity of the field generated by the object leads us to the same conclusion: the three dimensionality of the inner distribution cannot be determined by the two-dimensionality of the object manifestation, which is also coherent with the holographic principle. According to this, the maximal entropy contained in a limited space depends on the bounding surface and not on its volume (Susskind, 1997; Díaz, 2010). ${ }^{6}$

\section{The Nature of Perception}

If we now reckon the specificity of the animal sensitivity, we would encounter further limits concerning the amount of differences that a perceiving subject can acknowledge about the object. The more complex its sensitive organs are, the closer it can reach the stated limits. For instance, the eagle vision is closer to this boundary than what the human vision is (Díaz, 2008). But in the impression of the reality gathered by the subject there is an essential element which is consistent with our former conclusions: the differences encountered in sensation points to a radical incompleteness in relation to the reality which is being felt. As we have seen, there is an essential ambiguity regarding the possible volumetric configurations of the objects, as there is ambiguity in many illusory images considered in theory of perception (Rock, 1984). Although there is some kind of autonomy in perception with respect to the whole act of apprehending reality, this seems to be a

\footnotetext{
${ }^{6}$ As a corollary of the holographic principle, Bekenstein proposes that if the physics of our real (tetra-dimensional) universe were holographic, there would be an arbitrary set of physical laws which could be applied to some tri-dimensional space-time boundary. Therefore, there is a radical indeterminacy between this holographic universe -as he names it- and the physics we use to interpret it (Bekenstein, 2003).
} 
unitary act in which different sensitive structures take part (synchronic or diachronically) together with an intellective moment.

To illustrate this, let us consider a simple example. In fig. 3 , we try to deal with the ambiguity of the visual image of an object. It illustrates the dependence of complexity of percepts in perception. In (a) the simplest corresponding figure is perceived: a regular hole. However, in (b) because of geometrical regularity we may perceive a hole, but shading tends us to perceive an irregular protuberance. In fig. 3a, it is normally perceived a big square bump in whose middle there is a regular hole. Both the geometrical regularity and the shadowing drive us to perceive the bump with a hole. However, fig. $3 \mathrm{~b}$ brings us about the sensation of a more ambiguous object: the geometrical properties drive us to see again a hole which is differently colored than the rest. However, the shadowing invites us to see a smaller but irregular protuberance into the bump. According to Dretske's interpretation of perception, if we only assume informational relations holding the deterministic condition, we might say that we perceive in fig.3b a bump with a \{regular hole or an irregular protuberance\}. But this is not the case; we can alternatively perceive either an irregular protuberance or a regular and colored hole (different intellectual moments are taking part at each time, understood as different intellectual apprehensions of the object).
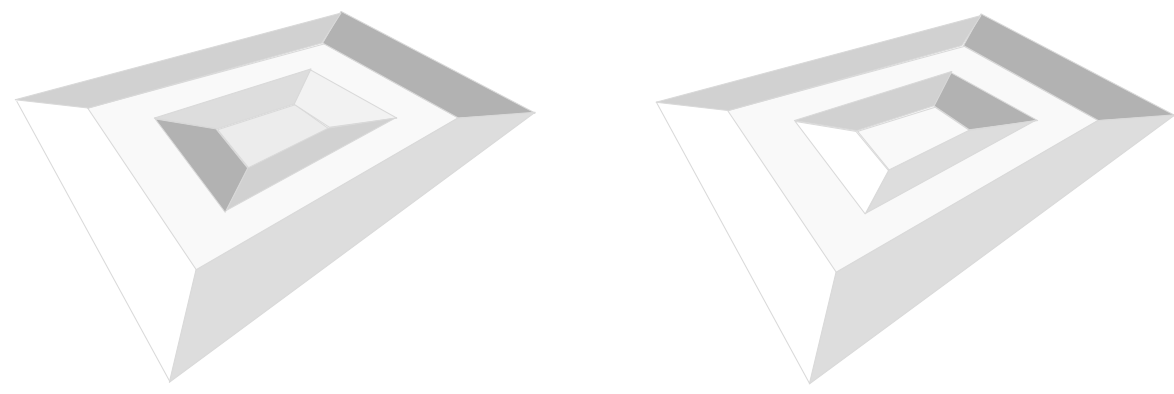

Figure 3: Perception of ambiguous figures.

Moreover, a different percept can also be obtained from fig.3a: a colored and irregular protuberance. Although this last percept is much lesser probable, it has been experimentally shown that, in spite of geometrical and color visual properties, the preferred visual percept might turn to be an irregular protuberance if the subject has the tactile sensation that something juts out (Robles-de-laTorre \& Hayward, 2001; Robles-de-la-Torre, 2006). Furthermore, even when the object is touched and the ambiguity -so to speak- is solved, the subject can visually perceive it as a hole, although (s)he is aware that a protuberance exists. This property allows us to speak of the aforementioned relative autonomy of perception (Rock, 1984), at the time that the awareness and intellection of reality forms some kind of unitary act in which different notes of reality, as well as different modes and structures of sensing take part.

As different experiments, carried out in the study of perception, have shown: the preferred perceptions tend to be those corresponding to the simplest configurations. For instance, in the previous example, a symmetrical bump, a homogeneous colored object, etc. It is as if perception would apply an Ockam's razor: if something admits a simpler description, then this is preferred. ${ }^{7}$

The more complex the sensitive structure, the greater the ambiguity of its perception and the more accurate the determination of the object. For instance, if we consider the simplest case of a cell, it has several means to sense the environment and to adapt to those variations which are relevant for its survival. More specifically, the unicellular organism Euglena viridis (among others of

\footnotetext{
${ }^{7}$ To this respect, it is relevant to mention that the mathematical regularization methods to solve inverse problems also appeal to this principle (Colton \& Krees, 1992).
} 
the same genus) has an eyespot apparatus which filters sunlight into the light-detecting, photosensitive structures at the base of its flagellum. This eyespot enables the cell to sense the strength and direction of light, and straightforward to move accordingly towards a medium of moderate light (away from darkness and bright light). ${ }^{8}$ The ambiguity of perception is here very low: the strength of light is high or low, and it comes from this or that direction. And it is also low the accuracy in the determination of the environmental state. In the animal vision, as we have shown before, the ambiguity can be much higher as it is the accuracy in the determination of the observed reality. Grasping more notes of reality, especially if they have different modality (e.g. visual and tactile notes) the ambiguity left by some partial perceptions (e.g. a visual percept) can decrease although new kinds of ambiguity may appear. Reality is more accurately sensed, feeling at the same time that the nonfelt part of reality is bigger. In our previous analysis of observation, sensation may grow in two dimensions, whereas the non-observed part is three-dimensional.

In other words, the constitutive indeterminacy of the manifestation of reality and the ambiguity of sensation (both closed related but not the same) bring about, on the one hand, the feeling that there is a part of reality beyond its manifestation; on the other hand, an invitation to find further notes to delve into the sensed reality. Probably if sensation were sufficient for a particular being in its interaction with its world, this invitation might not be felt. But in this case some kind of deterministic relation should provide univocal perceptions of what is being sensed allowing it to successfully deal with the perceived objects. This relation can be interpreted as a fixed assumed solution to the ambiguity of sensation. ${ }^{9}$ However, human sensation is clearly characterized by feeling the necessity of searching beyond the given sensations. The history of science as a whole could be interpreted in this sense, including deep changes in the sensed realities, as when the dawn star is beginning to be perceived as Venus, human as an evolved primate, atoms as something particularly empty, etc.

\section{References}

Bekenstein, J. D. (2003). Information in the holographic universe. Scientific American Magazine, 289(2), 58-65.

Colton, D. \& Krees, R. (1992). Inverse acoustic and electromagnetic scattering theory. Berlin: Springer.

Díaz Nafría, J. M. (2003). Contribución en métodos inversos para la caracterización de sistemas radiantes (Contributions in inverse methods for the characterization of radiant systems). Madrid, ES: Universidad Politécnica de Madrid. Retrieved from http://www.novatores.org/html/es/eprint/show.html?ePrintld=119

Díaz Nafría, J. M. (2008). The interminacy of observation. In J. M. Díaz \& F. Salto Alemany (Eds.) ¿Qué es información? (What is information?) Proceedings of the first International Meeting of Experts in Information theories: An interdisciplinar approach (pp. 489-502). León, ES: Universidad de León. Retrieved from http://wp.me/pzKNC-58.

Díaz Nafría, J. M. (2010). Holographic principle. In Díaz Nafría, J. M., Salto Alemany, F., \& Pérez-Montoro, M. (Eds.), Glossarium BITri: Glossary of concepts, metaphores, theories and problems regarding information. León, ES: Universidad de León. Retrieved from http://glossarium.bitrum.unileon.es/Home/logica-borrosa/fuzzy-logic.

Huygens, C. (1690). Treatise on light (S. P. Thompson, Transl.). Chicago, IL: University of Chicago Press. Retrieved from http://www.gutenberg.org/ebooks/14725.

Lemmermann, E. (1913). Eugleninae. In A. Pascher (Ed.), Die Süßwasser-Flora Deutschlands, Österreichs und der Schweiz (Vol. 2) (pp. 115-174). Jena: G. Fischer.

PEET. (2010). The Euglenoid project: Partnership for enhancing expertise in taxonomy-national science foundation. Retrieved from http://euglena.msu.edu.

Robles-De-La-Torre, G. \& Hayward, V. (2001). Force can overcome object geometry In the perception of shape through active touch. Nature, 412 (6845), 445-448.

Robles-De-La-Torre, G. (2006). The importance of the sense of touch in virtual and real environments. IEEE Multimedia 13(3), 24-30.

Rock, I. (1984). Perception. New York, NY: Scientific American Library.

\footnotetext{
${ }^{8}$ In the Euglena the afferent structures of the cell -sensing the environment- are directly connected to the efferent ones the flagellum which causes the necessary movement towards a more suitable environment (Lemmermann, 1913; PEET, 2010).

${ }^{9}$ Zubiri refers to this kind of apprehension as "apprehension of stimulation", characterized by a "formality of signitivity", which in evolutionary sense precedes the "formality of reality" (Zubiri, 1999).
} 
Schelkunoff, S. A. (1936). Some equivalence theorems of electromagnetics and their application to radiation problems. Bell System Technical Journal, 15, 92-112.

Shannon, C. E. (1949). Communication in the presence of noise. Proceedings of IRE, 37(1), 10-21.

Susskind, L. (1997, April). Black holes and the information paradox. Scientific American, 52-57.

Weisstein, E. W. (2008). Spherical harmonics. In Wolfram MathWorld. Retrieved from http://mathworld.wolfram.com/SphericalHarmonic.html.

Zubiri, X. (1999). Sentient intelligence (T. B. Fowler, Trans.). Washington, DC: Xavier Zubiri Foundation of North America..

\section{About the Authors}

José María Díaz Nafría

obtained M.Sc. in Telecommunication Engineering from the Universidad del País Vasco, Spain, and received his PhD in Telecommunication Engineering from the Universidad Politécnica de Madrid, Spain, with a dissertation on "Contributions to the electromagnetic inverse problem". He was also awarded with a M.A. in Philosophy by the Universidad Nacional de Educación a Distancia (UNED). He is currently visiting professor at the Munich University of Applied Sciences, investigator at the University of León and part of the board of directors of the Science of Information Institute, where he is also scientific advisor. He is member as well of several international scientific societies in the field of information theories.

He was research fellow at the Vienna University of Technology and at the Technical University of Madrid. He also served as professor at the University Alfonso X el Sabio in Madrid between 1997 and 2009. He has been visiting lecturer at the University of Furtwangen, Sankt Pölten University of Applied Sciences. Co-director of the "First International Meeting of Experts in Information Theories" (León, Spain, 2008) and the "Colloquium BITae" (León, Spain, 2009) currently coordinates an interdisciplinary research group meted around the BITrum project (Elucidation of the information concept) and co-edits with F. Salto and M. Pérez-Montoro the Glossarium BITri: glossary of concepts, metaphors, theories and problems concerning information (Universidad de León, 2010, http://glossarium.bitrum.unileon.es), where he authors several articles.

\section{Mario Pérez-Montoro Gutierrez}

Doctor in Philosophy and Education Sciences by the University of Barcelona, where he has been researcher at the Departamento de Lógica, Historia y Filosofía de la Ciencia. He studied in the Istituto di Discipline della Comunicazione in the Università di Bologna and was Visiting Scholar at the Center for the Study of Language and Information (CSLI) at Stanford University. He has been docent and researcher in several universities: Complutense de Madrid, Autònoma de Barcelona, Oberta de Catalunya and he is currently at the Department of Information Science at the University of Barcelona. One of his most known works is the book: The Phenomenon of Information (Scarecrow Press, 2007, published in Spanish by Trotta). He co-edits with J.M. Díaz and F. Salto the Glossarium BITri: glossary of concepts, metaphors, theories and problems concerning information (Universidad de León, 2010, http://glossarium.bitrum.unileon.es), where he authors several articles. 